\title{
Differential Impacts on Bacterial Composition and Abundance in Rhizosphere Compartments between Al-Tolerant and Al-Sensitive Soybean Genotypes in Acidic Soil
}

\author{
Zhong-Ling Wen ${ }^{1 \dagger}$, Min-Kai Yang ${ }^{1 \dagger}$, Aliya Fazal ${ }^{1 \dagger}$, Yong-Hui Liao ${ }^{1}$, Lin-Run Cheng ${ }^{2}$, \\ Xiao-Mei Hua ${ }^{3}$, Dong-Qing Hu${ }^{1}$, Ji-Sen Shi ${ }^{4}$, Rong-Wu Yang ${ }^{1}$, Gui-Hua Lu ${ }^{1,4}$, Jin-Liang Qi ${ }^{1,4 *}$, \\ Zhi Hong $^{1 *}$, Qiu-Ping Qian ${ }^{2 *}$, and Yong-Hua Yang ${ }^{1,4 *}$ \\ 'Institute for Plant Molecular Biology, State Key Laboratory of Pharmaceutical Biotechnology, School of Life \\ Sciences, Nanjing University, Nanjing 210023, P.R. China \\ ${ }^{2}$ Jinhua Academy of Agricultural Sciences, Jinhua 321017, P.R. China \\ ${ }^{3}$ Research Center for Soil Pollution Prevention and Control, Nanjing Institute of Environmental Sciences, MEE, \\ Nanjing 210042, P.R. China \\ ${ }^{4}$ Co-Innovation Center for Sustainable Forestry in Southern China, Nanjing Forestry University, Nanjing 210037, \\ P.R. China
}

Received: March 11, 2020 Accepted: May 15, 2020

First published online: May 20, 2020

*Corresponding authors Y.H.Y.

Phone/Fax: +86-25-89686305

E-mail: yangyh@nju.edu.cn Q.P.Q.

Phone: +86-25-89686305 E-mail: qianqiupingjh@163.com Z.H.

E-mail: zhihong@nju.edu.cn

J.L.Q.

E-mail: qijl@nju.edu.cn

${ }^{\dagger}$ These authors contributed equally to this work.

Supplementary data for this paper are available on-line only at http://jmb.or.kr.

pISSN 1017-7825 eISSN 1738-8872

Copyright(C) 2020 by The Korean Society for Microbiology and Biotechnology
In this study, two soybean genotypes, i.e., aluminum-tolerant Baxi 10 (BX10) and aluminumsensitive Bendi 2 (BD2), were used as plant materials and acidic red soil was used as growth medium. The soil layers from the inside to the outside of the root are: rhizospheric soil after washing (WRH), rhizospheric soil after brushing (BRH) and rhizospheric soil at two sides (SRH), respectively. The rhizosphere bacterial communities were analyzed by high-throughput sequencing of V4 hypervariable regions of $16 \mathrm{~S}$ rRNA gene amplicons via Illumina MiSeq. The results of alpha diversity analysis showed that the BRH and SRH of BX10 were significantly lower in community richness than that of BD2, while the WRH exhibited no significant difference between BX10 and BD2. Among the three sampling compartments of the same soybean genotype, WRH had the lowest community richness and diversity while showing the highest coverage. Beta diversity analysis results displayed no significant difference for any compartment between the two genotypes, or among the three different sampling compartments for any same soybean genotype. However, the relative abundance of major bacterial taxa, specifically nitrogen-fixing and/or aluminum-tolerant bacteria, was significantly different in the compartments of the BRH and/or SRH at phylum and genus levels, indicating genotype-dependent variations in rhizosphere bacterial communities. Strikingly, as compared with BRH and SRH, the WRH within the same genotype (BX10 or BD2) always had an enrichment effect on rhizosphere bacteria associated with nitrogen fixation.

Keywords: Soybean genotypes, rhizosphere compartments, bacterial communities, nitrogen fixation, aluminum tolerance

\section{Introduction}

Acidity in soil affects about $30 \%$ of the world's total land area and $50 \%$ of the world's potential arable land [1]. In South China, acidic red soil is a typical soil type with low $\mathrm{pH}$ value, low phosphorus content and high aluminum toxicity, which are directly related to restrictive crop growth and yield [2-4]. In order to adapt to the stressful conditions, plants can release root exudates such as organic acids, which can chelate toxic Al (aluminum) and mobilize P (phosphorus) [5-7]. Soybean is an important protein and oil source in the world and can also be utilized as an excellent rotation and intercropping crop [8]. Previous studies have shown that under severe P stress, $\mathrm{Al}$-tolerant soybean Baxi 10 (BX10) has a greater citrate efflux rate and nearly 2 times higher yield compared to Alsensitive soybean Bendi 2 (BD2) [9]. Moreover, the rhizosphere soil of BX10 exhibited changes in the ratio of gram-negative/gram-positive bacteria compared with BD2 [10].

Each plant species has its own specific rhizospheric bacterial community as different rhizosphere bacteria 
respond differently to different compounds of root exudates $[11,12]$. Previous studies have reported that plants can strongly influence the composition, structure, and activity of rhizosphere microbiota, especially with respect to the active populations [13-18]. Moreover, rhizosphere microorganisms such as bacteria, are closely related to plant health and growth $[13,19-21]$, thus leading to a complex binary relationship between plants and their rootassociated microorganisms. For example, experiments conducted by Li et al. showed that there are differences in the diversity and abundance of functional nifH gene (coding for nitrogenase iron protein) of the rhizosphere bacteria community at different growth stages between BX10 and BD2 [10, 22].

In this study, we aimed to elucidate the impacts of Al-tolerant (BX10) and Al-sensitive (BD2) soybean genotypes on the composition and abundance of rhizosphere bacterial communities at the flowering stage, among three different sampling compartments in acidic soil, via Illumina MiSeq sequencing platform.

\section{Materials and Methods}

Plant Materials, Sampling Methods and DNA Extraction

In this study, two soybean genotypes(Glycine max (L.) Merr.), BX10 (Baxi 10, aluminum (Al)-tolerance type) and BD2 (Bendi 2, Al-sensitive type), were selected as plant materials. Soybeans were planted in a rhizobox (rhizosphere box, $200 \mathrm{~mm}$ in length, $150 \mathrm{~mm}$ in width and $200 \mathrm{~mm}$ in depth) which was constructed with PVC material and divided into five parts by using a frame covered with nylon film, in order to prevent the root and root exudates from entering different soil compartments, but not water and nutrition $[23,24]$. Finely sieved acidic red soil ( $\mathrm{pH} 4.43)$ collected from the Ecological Experiment Station of Red Soil (28.208 N, 116.937 E) of the Chinese Academy of Sciences, Yingtan, Jiangxi Province, China [22], was evenly packed into five parts of the rhizobox while soybean was allowed to grow in the middle part.

Sampling at flowering stage was performed as described previously by $\mathrm{Li}$ et al. with some modifications $[25,26]$. The soil in the compartments on both sides of the rhizobox (rhizosphere soils between two partitions in rhizobox) was named SRH (rhizospheric soil at two sides) [24]. BRH (rhizospheric soil after brushing) samples were collected by brushing off the soil tightly adhering to the root surface, and then the WRH (rhizospheric soil after washing) samples were collected by centrifugation at 4,000 $\times g$ for $10 \mathrm{~min}$ after being washed with phosphatebuffered saline (PBS). Finally, all the samples were stored at $-80^{\circ} \mathrm{C}$ prior to DNA extraction.

Metagenomic DNA of every biological replicate was extracted from approximately $0.30 \mathrm{~g}$ of soil by using the PowerSoil DNA Isolation Kit (MoBio Laboratories Inc., USA). The experimental method followed the instructions with minor modifications $[27,28]$. After extraction, the quality of DNA samples was assessed on $1 \%$ agarose gel and quantified by using a Qubit Fluorometer (Qubit 2.0, Invitrogen, USA) to minimize the variability in surveys of bacterial communities [29].

\section{S rDNA Amplicon Sequencing and Analysis}

We used an improved dual-index high-throughput sequencing with paired-end 250nt and amplicons of approximately $290 \mathrm{bp}$ encompassing the V4 hypervariable region of the $16 \mathrm{~S}$ rDNA [30] using the following primers: forward primer 515F (5'-GTGCCAGCMGCCGCGGTAA-3') and reverse primer 806R (5'-GGACTA CHVGGGTWTCTAAT-3') [31]. The concentration of each qualified metagenomic DNA was tested and ensured to be more than $0.4 \mathrm{ng} / \mu \mathrm{l}$ [29]. PCR amplification, product purification, library quality determination and highthroughput sequencing of the qualified libraries on the Illumina MiSeq platform (Illumina, USA) with MiSeq Reagent Kit were conducted by BGI Tech Solutions Co., Ltd. (China). A total of 18 sequencing clean data has been submitted to the Sequence Read Archive (SRA) and the SRA accession number is PRJNA574806.

In order to characterize the bacterial community composition and structure in the SRH, BRH and WRH, highthroughput sequencing of $16 \mathrm{~S}$ rDNA amplicons using V4 region on the Illumina MiSeq platform was performed. Then, the clean tags were clustered into Operational Taxonomic Units (OTUs) with a 97\% similarity by using UPARSE software (v7.0.1090) [32] and the chimeras were filtered out by using UCHIME(v4.2.40) and 16S rDNA was screened for chimeras by mapping to a gold database (v20110519) [33]. The sub-sampling of OTU was performed using the software $\mathrm{R}$ (v3.1.3) in I-Sanger (http://www.i-sanger.com) according to the minimum sample number sequence. A total of 1,736,758 qualified paired-end reads with an average count of 96,486 (range: $80,603-114,407)$ per sample were obtained from 18 samples (Table S1). The high quality paired-end reads were then connected to tags based on 250 bp overlaps and a total of 1,214,537 tags were obtained (Table S1). After the removal of chimeras, clean tags were clustered into OTUs and a total of 35,651 OTUs were obtained (Table S1). The detailed information on OTUs with/without sub-sampling is summarized in Table S2 (Table S1). Then, the alpha and beta diversity analyses were conducted based on OTUs and species annotation results.

A rank-abundance curve was used to explain species abundance and evenness, and a Venn diagram was used to count the number of common and unique OTUs in multiple samples with $97 \%$ similarity level. Pan species were the sum of all species contained in all samples while core species were the number of common species in all the samples.

\footnotetext{
Alpha Diversity, Beta Diversity, Functional Prediction and Analysis, and Statistical Analysis

In order to analyze the complexity of species diversity in the environment, we used alpha diversity to reflect the community richness (Sobs, Chao, and Ace indices), community diversity (Shannon and Simpson indices) and community coverage (coverage index), while beta diversity analysis was calculated by using QIIME (v 1.8.0). We then evaluated differences of samples in species complexity by using different distance matrices [34, 35]. In this study, the results of three representative indices (Chao, Shannon, and Good's coverage) were shown to reflect the
} 
number of species, the uniformity of individual distribution in community and the coverage of all species, respectively.

Principal Component Analysis (PCA), a technique for analyzing and simplifying data sets using species abundances, was drawn by 'prcomp' in software R (v3.1.3). Principal Co-ordinates Analysis (PCoA) is a nonconstrained data dimensionality reduction analysis method, which can be used to find similarities or differences in sample community composition and is based on selected distance matrix (Bray-Curtis, weighted-UniFrac and unweighted-UniFrac). Both of these can identify potential principal components which can affect the diversity of sample community composition by dimensionality reduction. Unweighted Unifrac is affected by the number of rare OTUs in samples while Bray-Curtis and weighted Unifrac are based on the calculation of microbial evenness (the relative abundance of OTU), so the rare OTUs with low relative abundance have little impact on the results. Meanwhile, UniFrac distance matrices (including weighted Unifrac and unweighted Unifrac) are based on the comparison with phylogenetic tree, while other distance matrices like Bray-Curtis and Euclidean are based on the comparison without a phylogenetic tree. The PCoA was drawn by software R (v3.1.3) using vegan package. Nonmetric multidimensional scaling analysis (NMDS) is a data analysis method that simplifies the object of study (sample or variable), and NMDS can retain the original relationship between objects. NMDS involved the use of QIIME (v 1.8.0) to calculate the distance matrix and was also drawn by software R (v3.1.3) using vegan package. Partial Least Squares Discriminant Analysis (PLS-DA) effectively finds the influencing variables leading to the differences between groups by rotating the principal components. Unweighted Pair-group Method with Arithmetic Mean (UPGMA) is a clustering analysis method drawn by software R (v3.1.3) and was used to construct a tree structure to visualize the degree of difference in microbial evolution in different samples. Heatmaps aggregate species with high/low abundance and ours were drawn with the NMF package software $\mathrm{R}$ (v3.1.3). A ternary plot is an equilateral triangle used to compare three groups of samples and the proportion and relationship of different species in samples can be visually displayed and analyzed following methods by Bulgarelli et al. [36]. All these analyses were performed in platform I-Sanger (http://www.i-sanger.com). The COG function classification was also performed in platform I-Sanger and descriptive information and functional information of each COG can be parsed from eggNOG (evolutionary genealogy of genes: Non-supervised Orthologous Groups, http://eggnog.embl.de/) database while the abundance of each functional category can be calculated according to OTU abundance.

For statistical analysis, one-way ANOVA was used to evaluate the significance of samples in different alpha diversity indices. The analysis of similarities (ANOSIM) and PERMANOVA analysis (Adonis) were performed using vegan package of software R (v3.1.3) based on different distance metrics (Bray-Curtis, weighted-UniFrac and unweighted-UniFrac) by using databases Silva128/16s (SILVA database, http://www.arb-silva.de) [37].

\section{Results}

\section{Basic Data of Samples}

The rhizobox used in this study was designed as previously reported with minor modifications (Fig. 1) [10, 23]. The shape of the Rank-Abundance curve was gentle, and the curve also had a wide range on the horizontal axis. It shows that the abundance and evenness of 18 samples were relatively higher, and the trend of each sample was similar, which proves that the quality of samples and the depth and quality of sequencing were also relatively higher, indicating that the OTU coverage of the samples included sufficient detectable species in bacterial communities (Fig. S1). As shown in the Venn diagram, the OTU numbers of each sample were similar while the total number of shared OTUs of SRH, BRH and WRH was 1,227 (Fig. S2). According to the size of each list, the $\mathrm{BRH}$ samples have the most OTU numbers while the WRH samples have the fewest OTU numbers. Furthermore, as shown in Pan analysis and Core analysis, the number of core species (i.e., the number of species shared by all samples) is 331, while the total number of species contained by all samples is 5,829 (Fig. S3).

Comparative Analysis of the Alpha Diversity of Rhizosphere Bacterial Communities between BX10 and BD2

The rarefaction curves of alpha diversity indicated that the amount of sequencing data was enough to reflect the vast majority, and the community coverage was good. We therefore conducted comparative analysis of the alpha diversity through three different indices (Chao, Shannon, and Good's coverage). As shown in Fig. 2, for different

A

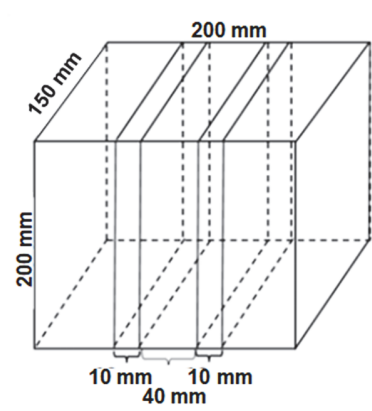

B

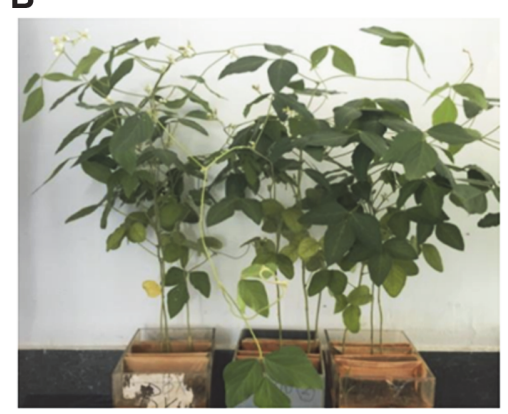

C

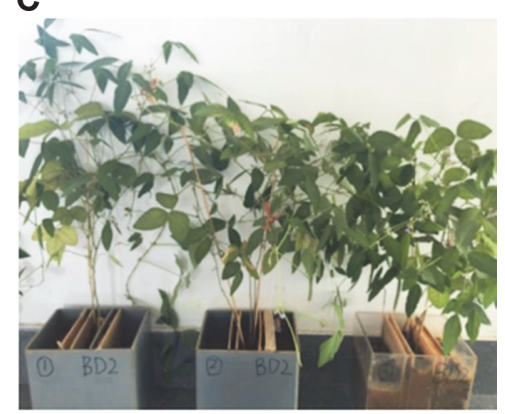

Fig. 1. Rhizobox diagram (A) and the rhizoboxes for soybean cultivation of BX10 (B) and BD2 (C). 
A

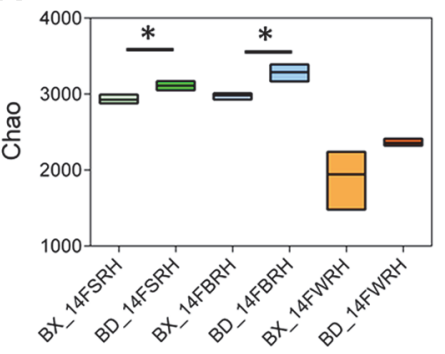

B

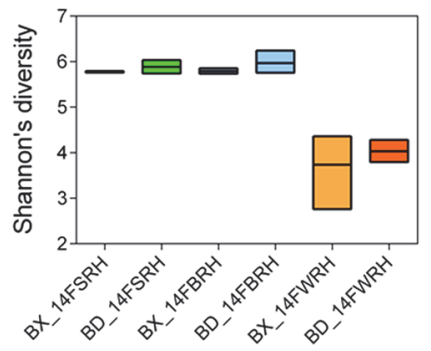

C

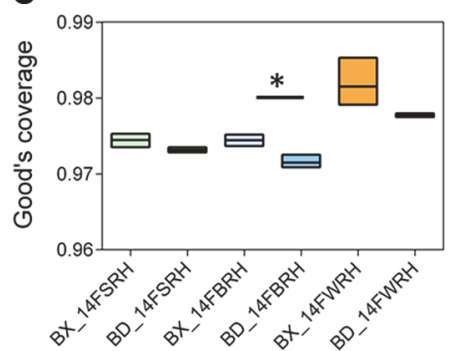

Fig. 2. The boxplot of alpha diversity of Chao index(A), Shannon index (B) and Good's coverage (C). SD represents standard deviation. Values were mean $\pm \mathrm{SD}(n=3)$. BX and $\mathrm{BD}$ represent the Al-tolerance soybean $\mathrm{BX} 10$ and $\mathrm{Al}-$ sensitive soybean $\mathrm{BD} 2$, respectively. F represents the flowering stages. SRH, BRH and WRH represent rhizospheric soil at two sides, rhizospheric soil after brushing, and rhizospheric soil after washing, respectively. The significance test method was performed using one-way ANOVA. The value in bold indicate the significant difference $(p<0.05)$ between the BX10 and BD2 groups by the tests.

sampling compartments of the same genotype (BX10 or BD2), the WRH has the lowest community richness and diversity, but meanwhile exhibited the highest coverage (Fig. 2). From the intergroup t-test of alpha diversity, we observed that, for WRH, there was no significant difference between the two genotypes in the six indices. However, the community richness in BRH was significantly lower in BX10 than that in BD2 (Fig. 2A), while community coverage in BX10 was higher than that in BD2 (Fig. 2C). Furthermore, SRH sample also showed significantly lower community richness in BX10 than that in BD2 (Fig. 2A).

Comparative Analysis of the Beta Diversity of Rhizosphere Bacterial Communities between BX10 and BD2

In order to study the similarities and differences in species composition and structure of different samples, a distance matrices and hierarchical clustering trees were constructed by using UPGMA. Cluster trees of beta diversity based on the Bray-Curtis distance (Fig. S4A), weighted-Unifrac distance (Fig. S4B) and unweightedUnifrac distance (Fig. S4C) showed that the samples from the same genotype of soybean and the same sampling compartment were clustered into one group. Furthermore, similar hierarchical clustering trees were obtained by three distance matrices above.

As shown in Fig. S5, samples from the same genotype or same sampling compartment (layer of soil) were clustered into one group via heat maps, which was consistent with the result of cluster trees shown above. Among different sampling compartments (layer of soil), high similarity of bacterial communities existed between BRH and WRH (Fig. S5), which has the same trend as the alpha diversity displaying richness of bacterial community (Fig. 2A).

Next, the PCA of OTU was done to examine the differences in the OTU composition between the SRH, BRH and WRH samples of BX10 and BD2 at the flowering stage (Fig. 3A). As shown in Fig. 3A, the samples from the same compartments (SRH, BRH or WRH) were clustered into one group, and there was no significant distinction among different soybean genotypes. Similar results were obtained from a PCoA chart based on Bray-Curtis (Fig. 3B). Similarly, the PCoA based on weighted-Unifrac distance and unweighted-Unifrac distance was consistent with the results based on Bray-Curtis (Fig. S6). As shown in Fig. S6, the rhizosphere bacterial

A

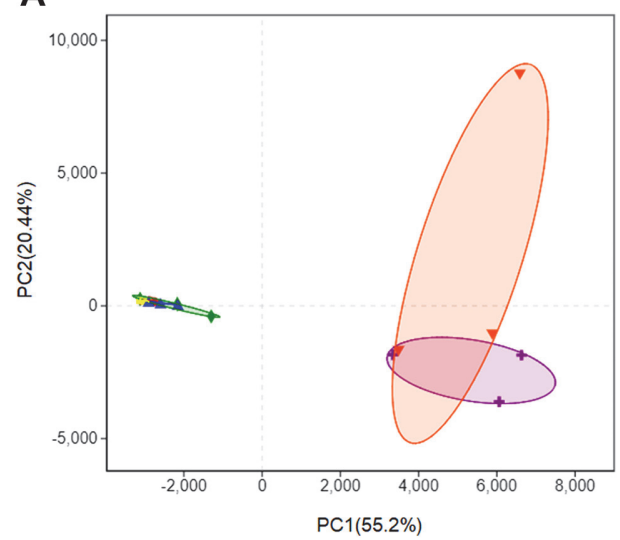

B

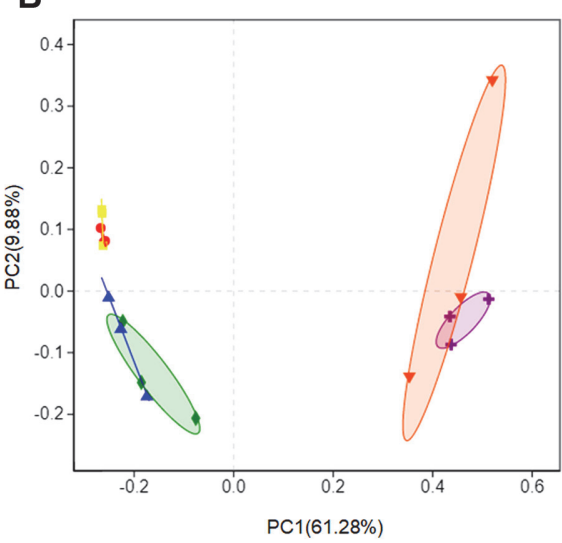

BD_14FBRH

BD_14FSRH

+BD_14FWRH

BX_14FBRH

$\triangle B X+14 F S R H$

$\nabla$ BX_14FWRH

Fig. 3. PCA based on OTU abundance of bacterial communities (A). PCoA based on Bray-Curtis distance of the rhizosphere bacterial communities between BX10 and BD2 (B). Treatment's details were as in Fig. 2. 
Table 1. Statistical analysis of bacterial community structure between the soybean line BX10 and BD2 with three different approaches.

\begin{tabular}{llcccc}
\hline \multirow{2}{*}{ Distance metrics } & \multicolumn{1}{c}{ Group vs. Group } & \multicolumn{2}{c}{ Adonis } & \multicolumn{2}{c}{ ANOSIM } \\
\cline { 3 - 5 } Bray-Curtis & & $\mathrm{R}^{2}$ & $p$-value & Statistic & $p$-value \\
& BX_14FSRH vs BD_14FSRH & 0.4380 & 0.1 & 0.7037 & 0.093 \\
& BX_14FBRH vs BD_14FBRH & 0.4949 & 0.1 & 0.7778 & 0.094 \\
\multirow{2}{*}{ weighted_Unifrac } & BX_14FWRH vs BD_14FWRH & 0.2225 & 0.3 & 0.0741 & 0.295 \\
& BX_14FSRH vs BD_14FSRH & 0.4924 & 0.1 & 0.5556 & 0.108 \\
unweighted_Unifrac & BX_14FBRH vs BD_14FBRH & 0.6024 & 0.1 & 0.7778 & 0.102 \\
& BX_14FWRH vs BD_14FWRH & 0.0736 & 0.9 & 0.1852 & 0.197 \\
& BX_14FSRH vs BD_14FSRH & 0.2348 & 0.1 & 0.8889 & 0.108 \\
& BX_14FBRH vs BD_14FBRH & 0.2776 & 0.1 & 1 & 0.095 \\
& BX_14FWRH vs BD_14FWRH & 0.2199 & 0.2 & 0.1481 & 0.302 \\
\hline
\end{tabular}

ANOSIM and Adonis based on the Bray-Curtis, weighted-Unifrac distance and unweighted-Unifrac distance metrics. The $p$ values $(p>0.05)$ indicated that no significant difference existed between the BX10 and BD2 groups by the tests. Treatment's details were as in Fig. 2.

communities from different compartments of BX10 were not distinct from those of BD2. The distance between $\mathrm{BRH}$ and SRH was close and indicated that high similarity of species composition existed between these two compartments. Furthermore, WRH was far away from BRH and SRH on the figures (Figs. 3 and S6).

We next performed ANOSIM and Adonis analysis of bacterial communities based on different distance metrics. The results of ANOSIM and Adonis displayed no significant difference between the beta diversity of rhizosphere bacterial communities of BX10 and BD2 ( $p$-value $>0.05$ ) (Table 1). Moreover, the ANOSIM and Adonis analysis of bacterial community structure between different sampling compartments based on the BrayCurtis matrix indicated no significant difference between SRH and BRH, SRH and WRH, BRH and WRH ( $p$ value $>0.05$ ) (Table S3).

As shown in Fig. S7, samples from the same sampling compartments clustered into one group while there also existed distinction in distance between different sampling compartments via NMDS. Similarly, samples were clustered by different sampling compartments and soybean genotypes via PLS-DA (Fig. 4).

\section{Comparison of the Composition of the Major Bacterial Taxa}

The results of taxonomic analysis helped us identify the community structure and composition of different samples at different taxonomic levels (phylum, class, order, family, genus and species). In this way, the information of composition and abundance for each sample could be presented intuitively. The results of ternary plots were shown in Fig. S8. The relative abundance and proportional contribution of OTUs in three compartments indicated that Psedumonas and Pseudarthrobacteroxydans, which belong to phyla Proteobacteria and Actinobacteria, have the highest abundance, while Candidatus Nitrosotalea, which belong to phylum Tenericutes, has the lowest abundance in WRH compared to BRH and SRH.

The taxonomic composition distribution histogram of each sample was displayed at phylum, class, order, family, genus and species level (Fig. S9). Then we compared the eight major phyla in each sample. As shown in Table 2, the most abundant phylum was Acidobacteria followed by Proteobacteria, Chloroflexi, and Thaumarchaeotain in SRH and BRH. However, in WRH, the most abundant phylum was Proteobacteria followed by Actinobacteria and Firmicutes.

As shown in Table 2, Figs. 5 and S9, for the same sampling compartment, the relative abundances of Proteobacteria in BX_14FSRH, Chloroflexi in BX_14FBRH and Actinobacteria in BX_14FBRH were

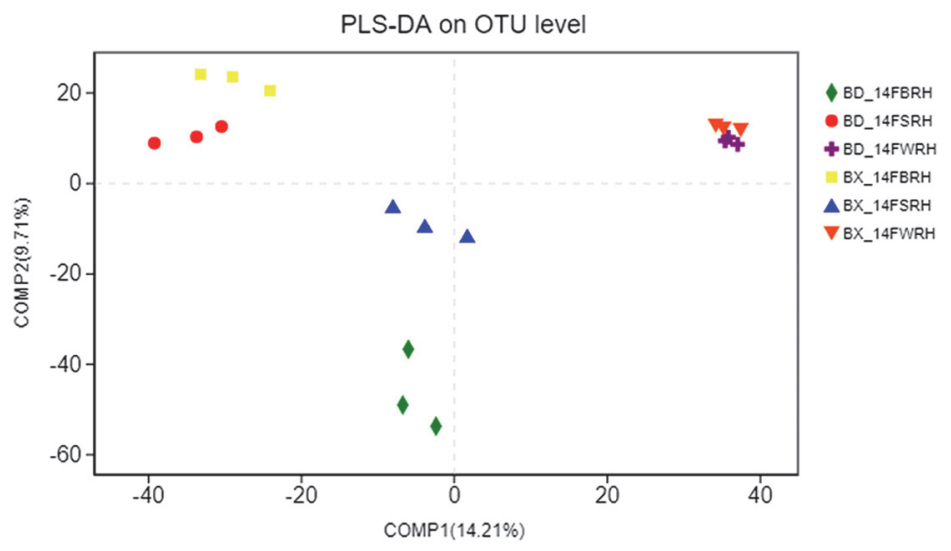

Fig. 4. PLS-DA on OTU level of the rhizosphere bacterial communities between BX10 and BD2. Treatment's details were as in Fig. 2. 
Table 2. The relative abundance of eight major phyla in each sample (\%).

\begin{tabular}{|c|c|c|c|c|c|c|c|c|}
\hline Group & Proteobacteria & Acidobacteria & Chloroflexi & Actinobacteria & Bacteroidetes & Verrucomicrobia & Firmicutes & Thaumarchaeota \\
\hline BD_14FSRH & $13.04 \pm 1.39$ & $25.55 \pm 4.56$ & $14.89 \pm 0.39$ & $4.22 \pm 0.09$ & $3.74 \pm 1.06$ & $4.92 \pm 1.09$ & $1.76 \pm 0.18$ & $15.08 \pm 1.37$ \\
\hline BX_14FSRH & $25.23 \pm 4.07$ & $24.08 \pm 3.87$ & $9.86 \pm 1.63$ & $3.39 \pm 0.73$ & $7.46 \pm 4.03$ & $4.42 \pm 0.29$ & $1.84 \pm 0.24$ & $11.51 \pm 4.28$ \\
\hline BD_14FBRH & $26.16 \pm 1.69$ & $20.36 \pm 1.86$ & $9.03 \pm 2.37$ & $2.85 \pm 0.46$ & $7.97 \pm 1.25$ & $4.61 \pm 0.50$ & $6.21 \pm 7.08$ & $10.99 \pm 6.80$ \\
\hline BX_14FBRH & $13.24 \pm 2.00$ & $26.55 \pm 3.74$ & $14.87 \pm 2.12$ & $4.73 \pm 0.61$ & $2.31 \pm 0.50$ & $4.77 \pm 0.81$ & $2.21 \pm 0.25$ & $16.75 \pm 1.10$ \\
\hline BD_14FWRH & $44.89 \pm 4.59$ & $5.92 \pm 1.57$ & $3.59 \pm 0.56$ & $18.76 \pm 5.09$ & $4.20 \pm 2.85$ & $1.84 \pm 0.17$ & $17.20 \pm 4.56$ & $0.24 \pm 0.10$ \\
\hline BX_14FWRH & $41.64 \pm 25.51$ & $5.54 \pm 3.30$ & $3.71 \pm 2.04$ & $24.12 \pm 7.64$ & $4.62 \pm 2.55$ & $1.25 \pm 0.62$ & $15.71 \pm 15.18$ & $0.25 \pm 0.18$ \\
\hline
\end{tabular}

SD represents standard deviation. Values were mean $\pm \mathrm{SD}(n=3)$. The values in bold indicate the significant difference $\left[p<0.05\left({ }^{*}\right)\right]$ between the $\mathrm{BX} 10$ and $\mathrm{BD} 2$ groups according to Student's t-test. Treatment's details were as in Fig. 2.

significantly higher than that in BD2, meanwhile the Proteobacteria in BX_14FBRH, Chloroflexi in BX_14FSRH and Bacteroidetes in BX_14FBRH were significantly lower than that in BD2. Further analysis of the differences in bacteria composition on genus level indicated that BX_14FSRH was enriched for Burkholderia (belonging to phylum Proteobacteria) and depleted for Ktedonobacter (belonging to Chloroflexi). Similarly, BX_14FSRH was enriched for Ktedonobacter and Acidothermus (belonging to Actinobacteria), while depleted forBurkholderia and Mucilaginibacter (belonging to Bacteroidetes), as compared to the same sampling compartment in BD2. Furthermore, the species composition and abundance of WRH exhibited no significant different between BX10 and BD2. Moreover, the species composition and abundance of WRH were different from those of SRH and BRH at different taxonomic levels of the same soybean genotypes (BX10 or BD2). Comparing the community classification and abundance of samples at phylum level, the relative abundance of phyla Proteobacteria, Actinobacteria and Firmicutes in WRH were significantly higher than that in SRH and BRH (Fig. 3).

Next, we further compared the composition of the major bacterial taxa at the class, order or family levels (Fig. S9), especially the difference between WRH and the other two compartments. At class level, the relative abundances of Bacilli, Gammaproteobacteria and Actinobacteria in the WRH were significantly higher than that in SRH and BRH. At order level, the relative abundances of Bacillales, Pseudomonadales and Micrococcales in the WRH were significantly higher than that in SRH and BRH. At family level, the relative abundances of Bacillacea, Paenibacillacea, Pseudomonadaceae and Micrococcaceae in the WRH were significantly higher than that in SRH and BRH. At genus level, the relative abundances of Bacillus, Paenibacillus, Pseudomonas and Pseudarthrobacter in the WRH were significantly higher than that in SRH and BRH. At species level, the relative abundances of Paenibacillusodorifer, Paenibacilluscastaneae, Paenibacilluspectinilyticus, Pseudomonas brassicacearum and Pseudarthrobacteroxydans in the WRH were significantly higher than that in SRH and BRH.

\section{Functional Prediction and Analysis of 16S rDNA}

Functional prediction of $16 \mathrm{~S}$ rDNA was conducted by standardizing OTU abundance using the software PICRUSt, and then obtaining COG family information corresponding to OTU by Greengene ID, and calculating the COG abundance of each sample. As shown in Fig. 6A, the composition and abundance of COG function classification of each sample was consistent with the $16 \mathrm{~S}$ functional prediction. We therefore concluded the relative abundances of some main COG functional classes (Fig. 6B), and found that the functional OTUs related to amino acid transport and metabolism, cell wall/ membrane/ envelope biogenesis, signal transduction mechanism, energy production and conversion, carbohydrate transport and metabolism, transcription, inorganic ion transport and metabolism, replication, and recombination and repair were relatively higher in abundance (abundance $>2,000,000$ ).

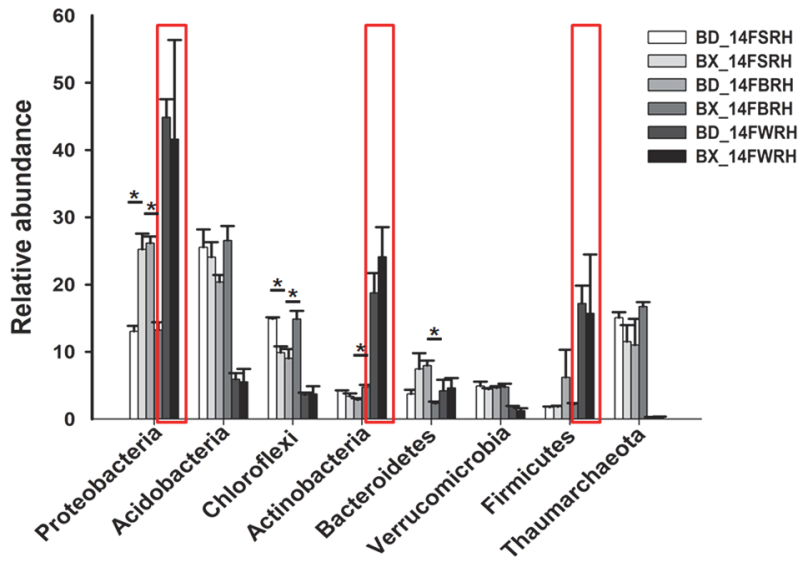

Fig. 5. Relative abundances of eight major phyla in each sample. The relative abundances of phyla Proteobacteria, Actinobacteria and Firmicutes in WRH were significantly higher than that in SRH and BRH and were marked in red boxes. Asterisk $\left({ }^{*} p<0.05\right)$ indicates significant difference according to Student's $t$-test. Treatment's details were as in Fig. 2. 
A

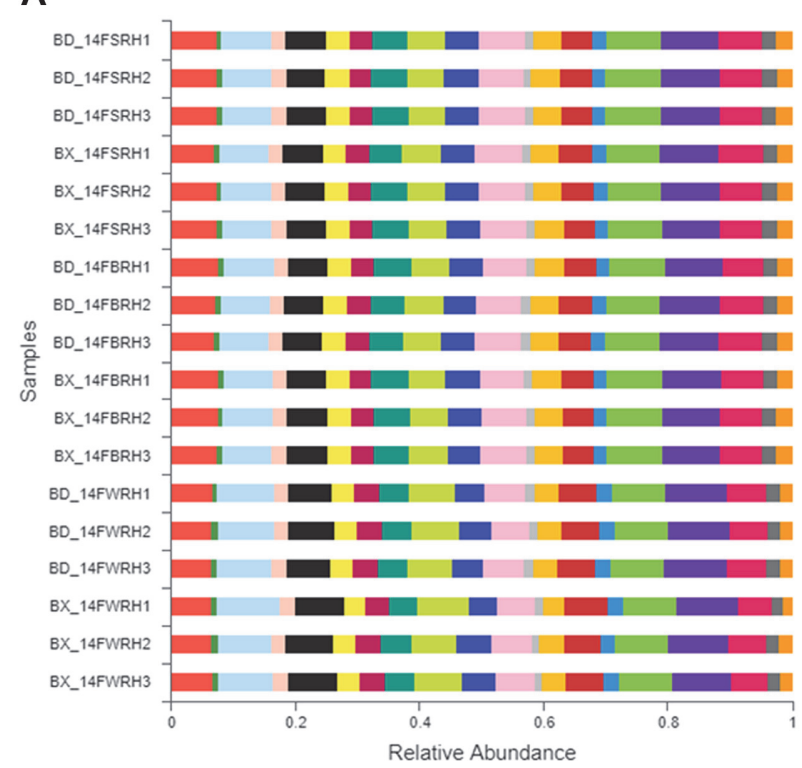

COG function classification

A : RNA processing and modification B : Chromatin structure and dynamics C : Energy production and conversion D : Cell cycle control, cell division, chromosome partitioning E : Amino acid transport and metabolism IIf $\mathrm{F}$ : Nucleotide transport and metabolism G : Carbohydrate transport and metabolism $\mathrm{H}$ : Coenzyme transport and metabolism I: Lipid transport and metabolism

$\mathrm{J}$ : Translation, ribosomal structure and biogenesis $\mathrm{K}:$ Transcription

$\mathrm{L}$ : Replication, recombination and repair $\mathrm{M}$ : Cell wall/membrane/envelope biogenesis $\mathrm{N}$ : Cell motility

0 : Posttranslational modification, protein turnover, chaperones D : Inorganic ion transport and metabolism

Q: Secondary metabolites biosynthesis, transport and catabolism $\mathrm{R}$ : General function prediction only

S : Function unknown

$\mathrm{T}$ : Signal transduction mechanisms

U : Intracellular trafficking, secretion, and vesicular transport $\mathrm{v}$ : Defense mechanisms W: Extracellular structures Y: Nuclear structure $\mathrm{z}$ : Cytoskeleton

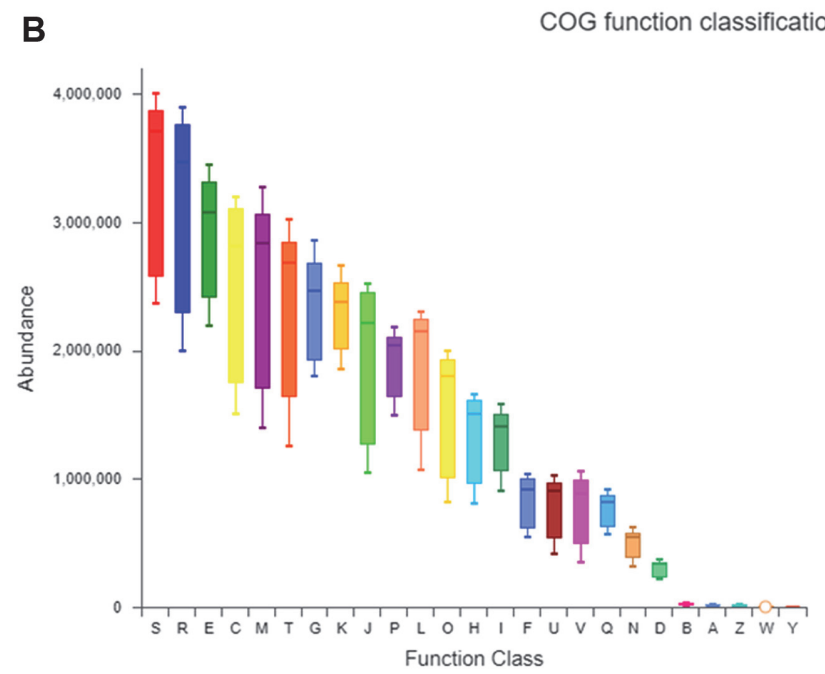

R:General function prediction only

E:Amino acid transport and metabolism

C:Energy production and conversion

M:Cell wall/membrane/envelope biogenesis

T:Signal transduction mechanisms

G:Carbohydrate transport and metabolism

K:Transcription

$\mathrm{J}$ :Translation, ribosomal structure and biogenesis

P:Inorganic ion transport and metabolism

L:Replication, recombination and repair

O:Posttranslational modification, protein turnover, chaperones

$\mathrm{H}:$ Coenzyme transport and metabolism

I:Lipid transport and metabolism

F:Nucleotide transport and metabolism

U:Intracellular trafficking, secretion, and vesicular transport

v:Defense mechanisms

Q:Secondary metabolites biosynthesis, transport and catabolism

$\mathrm{N}$ :Cell motility

D:Cell cycle control, cell division, chromosome partitioning

B:Chromatin structure and dynamics

A:RNA processing and modification

Z:Cytoskeleton

W:Extracellular structures

Function Class

Fig. 6. COG functional prediction. Treatment's details were as in Fig. 2.

According to the relative abundance of COG functional classification, we found that five COGs, i.e., COG1348, COG5420, COG5554, COG5456 and COG4656, are directly related to nitrogen-fixation (Table S4). Then, the statistics were analyzed by using one-way ANOVA (Table 3). As shown in Table 3, the relative abundances of COG5554, COG5456 and COG4656 were significantly lower in BX_14FBRH than that in BD_14FBRH. Moreover, the relative abundance of COG5554 was significantly lower in BX_14FSRH than that in BD_14FSRH.

Table 3. Statistics of COG function classification related to nitrogen-fixation or aluminum resistance.

\begin{tabular}{|c|c|c|c|c|c|c|}
\hline & $\begin{array}{c}\text { BX_14F } \\
\text { SRH }\end{array}$ & $\begin{array}{c}\text { BD_14F } \\
\text { SRH }\end{array}$ & $\begin{array}{c}\text { BX_14F } \\
\text { BRH }\end{array}$ & $\begin{array}{c}\text { BD_14F } \\
\text { BRH }\end{array}$ & $\begin{array}{c}\text { BX_14F } \\
\text { WRH }\end{array}$ & $\begin{array}{c}\text { BD_14F } \\
\text { WRH }\end{array}$ \\
\hline COG1348 & 1968.67 & 2328.67 & 1877.33 & 2270.33 & 1314.33 & 1059.67 \\
\hline COG5420 & 132.33 & 131.33 & 129.67 & 204.67 & 142.67 & 185 \\
\hline COG5554 & 181 & 143.33 & 136.67 & 263.67 & 871.67 & 422.33 \\
\hline COG5456 & 450.333 & 351 & 346.67 & 575.33 & 459.67 & 392.67 \\
\hline COG4656 & 1079 & 1321.67 & 1207.67 & 1354.33 & 1047 & 732.33 \\
\hline COG4100 & 534.67 & 541 & 365.67 & 578.67 & 479.67 & 529.67 \\
\hline
\end{tabular}

Values were mean of 3 replications. Treatment's details were as in Fig. 2. The significance test method was performed using oneway ANOVA. The values in bold indicate the significant difference $(p<0.05)$ between the BX10 and BD2 groups by the tests. 
The three COGs, i.e., COG5554, COG5456 and COG4656, were described as nitrogen fixation protein, nitrogen fixation protein $\mathrm{FixH}$, and required for nitrogen fixation, which may be part of a membrane complex functioning as an intermediate in the electron transport to nitrogenase, respectively. Furthermore, when comparing these five COG function classifications in the same soybean genotype (BD2 or BX10) among the three sampling compartments, the COG5554 was higher in WRH than in SRH and BRH, while the COG4656 was lower in WRH than in SRH and BRH in BD2.

We then compared the relative abundance of functional genes related to aluminum resistance in the same compartment between the two soybean genotypes and found that COG4100, which was recognized as Alresistance protein, had no significant difference between BX10 and BD2 in any compartments. Similarly, the statistics for COG4100 also showed no significant difference among the three different compartments of the same soybean genotype.

\section{Discussion}

In this study, we provided an overall framework through comparison of the previous opinions $[24,38]$ on the concept of rhizosphere microbiota with modifications [26, 28]. Different layers of soil were divided into three types, SRH, BRH and WRH. The SRH compartment was separated by a nylon net, the plant roots could not enter other sections although root exudates could invade the soil, meaning that the soil of other compartments may also be affected by the plants. We then aimed to find the effects of the different soybean genotypes on the rhizosphere bacteria community and to explore the relationship between aluminum-tolerance and root bacterial community in plants.

Analysis of alpha diversity of rhizosphere bacterial communities between BX10 and BD2 showed that SRH from BX10 had significantly lower community richness, while the BRH showed significantly lower community richness and higher community coverage in BX10 than that in BD2. The alpha diversity of WRH had no significant difference between the two genotypes. However, compared with SRH and BRH, the WRH had the lowest community richness and diversity along with the highest coverage. From the results above, we conclude that soybean genotypes and sampling compartments exerted some influence on the alpha diversity of rhizosphere bacteria, where the genotype BX10 and the sampling compartment WRH had a significantly lower species richness. The previous studies indicated that the BX10 and BD2 exhibited distinct rhizosphere microbial communities [10], and differences existed in the community structure between BX10 and BD2 at different sampling stages [22]. We compared the beta diversity of rhizosphere bacterial communities between BX10 and BD2. ANOSIM and Adonis analysis were used to assess the variations in bacterial rhizosphere diversity between BX10 and BD2 groups, and the results showed no significant difference. It was reported that uncultured Acidobacterium, Chloroflexi, and actinomycete enriched in BD2, and BD2 was affiliated with Rhizobium sp. and Azospirillumbrasilense, which have the potential to promote plant growth $[10,22]$. Thus, we compared the composition of the major bacterial taxa to find out the difference in the composition of rhizosphere bacterial community between BX10 and BD2. For the same sampling compartment, some significant difference in community classification and abundance existed between the samples from two genotypes of soybean, for example, the SRH of BX10 was enriched for Burkholderia and depleted for Ktedonobacter as compared with BD2. On the contrary, the BRH of BX10 was enriched for Ktedonobacter and depleted for Burkholderia. These two genera are reported to be associated with nitrogen-fixation $[39,40]$. As compared to BD2, the genus Burkholderia in BX10 was enriched in SRH while depleted in BRH. Previous studies have proved that some species of Burkholderia are aluminum-tolerant, so the distribution of Al-tolerant bacteria in different root compartments of different genotypes of soybean in acidic red soil is a subject worthy of further attention [41]. Root exudation of organic acids is the main mechanism of aluminum-tolerance in plants as they cause Al-detoxification by their Alchelating ability. In our study, the reason for the differences in bacterial communities corroborate with the previous studies where, two soybean cultivars (Al-tolerant BX10 and Al-sensitive $\mathrm{BD} 2$ ) responsive to Al-stress have different root exudate compositions that resulted in a variable diazotrophic community $[22,23]$.

The results of cluster trees and heatmaps showed that, the samples from one genotypes of soybean and from one sampling compartment were clustered into one group. Moreover, WRH was clustered far away from BRH and SRH according to the results of PCA, PCoA and PLS-DA, indicating that differences may exist in species composition between them. However, the results of ANOSIM and Adonis (Table S3) indicated that no significant difference existed among the three sampling compartments. When comparing the composition of the major bacterial taxa for the same genotype among the three sampling compartments, we found that the three major phyla Proteobacteria, Actinobacteria and Firmicutes, were significantly higher in the WRH than that in SRH and BRH (Table 2, Figs. 5, S8, and S9). Among them, we focused on the composition of nitrogen-fixing bacteria. The results of ternary plots showed that Psedumonas and Pseudarthrobacteroxydans, which belong to phyla Proteobacteria and Actinobacteria, had the highest abundance in WRH. The relative abundances of some major bacterial taxa were significantly higher in WRH than that in SRH and BRH. For example, some of the species of Bacillus belong to a plant growth-promoting bacteria (PGPB) class. The species Paenibacillusodorifer, Paenibacilluscastaneae and Paenibacillus pectinilyticus belong to the genus Paenibacillus, which was originally included within the genus Bacillus and reclassified as a separate genus in 1993 [42], and has been detected in a variety of environments including soil, water, rhizosphere, vegetable matter, and so on [43-45]. P. odorifer and $P$. castaneae belong to the nitrogen-fixing bacteria [46, 47]. Pseudomonas brassicacearum belong to the genus Pseudomonas and gets its name because it the roots of Brassica napus [48], and P. brassicacearum exhibits both pathogenic and growth-promoting properties in its interaction with tomato [49]. The species Pseudarthrobacteroxydans belongs to the genus Pseudarthrobacter, which is a new genus from the original genus 
Arthrobacter, so P. oxydans is also known as Arthrobacteroxydans. The model strain Arthrobacteroxydans DSM20119 T (GenBank: X83408) was reported to have the highest sequence similarity (99.132\%) with a nitrogen-fixing bacterium with ACC deaminase activity [50]. In summary, the rhizosphere bacteria associated with nitrogenfixation were significantly higher in WRH than that in SRH and BRH. Thus, we can tentatively draw conclusions that the distance from the sampling compartments to the roots can dramatically impact the species composition of rhizosphere bacterial communities, especially those associated with nitrogen-fixation.

Previous studies reported that the rhizosphere show enrichment processes in rhizosphere microbes with particular functional genes [51]. Thus, we compared the COG function classification and the relative abundance of COG functional classification. Functional prediction analysis of $16 \mathrm{~S}$ rDNA gene showed that the relative abundances of three special COGs; COG5554, COG5456, and COG4656, were significantly lower in BX_14FBRH than that in BD_14FBRH, while the relative abundance of COG5554 was significantly lower in BX_14FSRH than that in BD_14FSRH. The three COGs are related to nitrogen-fixation, indicating that the genotypes of soybean have impacts on rhizosphere bacterial communities, especially on microbes with particular functional genes associated with nitrogen-fixation. The WRH of BD2 was also higher in COG5554 and lower in COG4656 when compared among the three sampling compartments. These results are consistent with the results of composition of the major bacterial taxa. Major differences existed in the abundance and dominance of rhizosphere microorganisms, which is directly related to the changes in the composition of exudates released by plant roots. The difference in the abundance of nitrogen-fixing genes between BX10 and BD2 is possibly due to differential composition of the exudates secreted by the roots, as it is well known that there are differences in organic acid secretion between the two soybean cultivars under aluminum stress in acidic soil [22, 23]. On the other hand, the role of nitrogen-fixing genes in the soil is supposed to be directly related to the activity of nitrogenfixing bacterial communities. Once rhizosphere nitrogen-fixing bacteria are enriched, they would contribute to the growth of host plants by fixing atmospheric nitrogen into nitrates that are then taken up by plants, and also to help enhance the host tolerance to aluminum stress [13, 19-21]. In turn, host plants with better growth under aluminum stress might secrete more root exudates to the rhizosphere, which would help alleviate the aluminum toxicity in soils, and meanwhile, would further shape the composition and diversity of soil microbial communities [11-18].

Because the DNA extraction and sequencing was carried out earlier in this paper, the primers we used were $515 f$ Original and 806r Original as proposed by Caporaso et al. in 2012 [31]. Subsequently, Parada et al. and Apprill et al. proved that the $515 \mathrm{f}$ Modified and 806r Modified not only corrected the detection of bacterial communities, but also facilitated the detection of archaeological communities, which has been widely used nowadays [52-55].

In conclusion, in the comparison of alpha diversity, the BRH and SRH of BX10 were significantly lower on community richness than that of BD2, while the WRH showed no significant difference between BX10 and BD2. Among the three sampling compartments of the same soybean genotype (BX10 or BD2), WRH had the lowest community richness and diversity while showed the highest coverage. No significant difference in the overall rhizosphere bacterial communities existed for any compartment between the two genotypes in the comparison of beta diversity. However, compared with BD2, the SRH of BX10 was enriched for Proteobacteria (especially in the genus Burkholderia), while BRH of BX10 depleted Proteobacteria. The genus Burkholderia is reported to be associated with nitrogen-fixation and also contains bacterial species with aluminum-tolerance. In our study, no significant differences in beta diversity and in the relative abundances of major bacterial taxa were found at phylum level of WRH between BX10 and BD2. However, as compared with BRH and SRH, WRH across the same genotype (BX10 or BD2) always had an enrichment effect on rhizosphere bacteria associated with nitrogenfixation. Lastly, the relative abundance of COG function classification also showed that BD2 exerted enrichment effects on some COG functional genes that were directly related to nitrogen-fixation, while there were no significant difference in the relative abundance of functional genes related to aluminum resistance between BX10 and BD2.

Finally, our results demonstrate that the genotype of soybean dramatically affected the rhizosphere communities, especially those associated with nitrogen- fixation and/or aluminum-tolerance in the compartments of the BRH and/or SRH, but not in the WRH. However, WRH had the highest abundance of nitrogen-fixing bacteria compared to the two compartments across the two genotypes. The interaction of rhizosphere microbiome and plants is a complex phenomenon which needs to be explored in detail. Overall, our results indicate that soybean genotype and sampling compartments had differential impacts on the composition and structure of rhizosphere communities.

\section{Acknowledgments}

This research was supported by the National Key Research and Development Program of China (2016YFD0101005), the National Natural Science Foundation of China (31870495, 31271744 and 31372140), the National Important Science \& Technology Specific Project (2016ZX08011-003), and the Program for Changjiang Scholars and Innovative Research Team in University (IRT_14R27).

\section{Conflict of Interest}

The authors have no financial conflicts of interest to declare. 


\section{Reference}

1. Vonuexkull HR, Mutert E. 1995. Global extent, development and economic-impact of acid soils. Plant Soil 171: 1-15.

2. Ryan PR, Shaff JE, Kochian LV. 1992. Aluminum toxicity in roots - Correlation among ionic currents, ion fluxes, and root elongation in aluminum-sensitive and aluminum-tolerant wheat cultivars. Plant Physiol. 99: 1193-1200.

3. Koyama H, Toda T, Yokota S, Dawair Z, Hara T. 1995. Effects of aluminum and Ph on root-growth and cell viability in ArabidopsisThaliana strain landsberg in hydroponic culture. Plant Cell Physiol. 36: 201-205.

4. Kochian LV. 1995. Cellular mechanisms of aluminum toxicity and resistance in plants. Annu. Rev. Plant Phys. 46: 237-260.

5. Ryan PR, Delhaize E, Jones DL. 2001. Function and mechanism of organic anion exudation from plant roots. Annu. Rev. Plant Phys. 52: 527-560.

6. Wei LL, Chen CR, Xu ZH. 2010. Citric acid enhances the mobilization of organic phosphorus in subtropical and tropical forest soils. Biol. Fertil. Soils 46: 765-769.

7. Ma JF, Ryan PR, Delhaize E. 2001. Aluminium tolerance in plants and the complexing role of organic acids. Trends Plant Sci. 6: 273278.

8. Cheng FX, Cao GQ, Wang XR, Zhao J, Yan XL, Liao H. 2009. Isolation and application of effective nitrogen fixation rhizobial strains on low-phosphorus acid soils in South China. Chinese Sci. Bull. 54: 412-420.

9. Dong DF, Peng XX, Yan XL. 2004. Organic acid exudation induced by phosphorus deficiency and/or aluminium toxicity in two contrasting soybean genotypes. Physiol. Plant. 122: 190-199.

10. Yang TY, Liu GL, Li YC, Zhu SM, Zou AL, Qi JL, et al. 2012. Rhizosphere microbial communities and organic acids secreted by aluminum-tolerant and aluminum-sensitive soybean in acid soil. Biol. Fertil. Soils 48: 97-108.

11. Jaeger CH, Lindow SE, Miller S, Clark E, Firestone MK. 1999. Mapping of sugar and amino acid availability in soil around roots with bacterial sensors of sucrose and Tryptophan. Appl. Environ. Microbiol. 65: 2685-2690.

12. Berg G, Eberl L, Hartmann A. 2005. The rhizosphere as a reservoir for opportunistic human pathogenic bacteria. Environ. Microbiol. 7: 1673-1685.

13. Bulgarelli D, Schlaeppi K, Spaepen S, van Themaat EVL, Schulze-Lefert P. 2013. Structure and functions of the bacterial microbiota of plants. Annu. Rev. Plant Biol. 64: 807-838.

14. Bais HP, Weir TL, Perry LG, Gilroy S, Vivanco JM. 2006. The role of root exudates in rhizosphere interations with plants and other organisms. Annu. Rev. Plant Biol. 57: 233-266.

15. Paterson E, Gebbing T, Abel C, Sim A, Telfer G. 2007. Rhizodeposition shapes rhizosphere microbial community structure in organic soil. New Phytol. 173: 600-610.

16. Aira M, Gomez-Brandon M, Lazcano C, Baath E, Dominguez J. 2010. Plant genotype strongly modifies the structure and growth of maize rhizosphere microbial communities. Soil Biol. Biochem. 42: 2276-2281.

17. Kondorosi E, Mergaert P, Kereszt A. 2013. A Paradigm for endosymbiotic life: Cell differentiation of rhizobium bacteria provoked by host plant factors. Annu. Rev. Microbiol. 67: 611-628.

18. Ofek M, Voronov-Goldman M, Hadar Y, Minz D. 2014. Host signature effect on plant root-associated microbiomes revealed through analyses of resident vs. active communities. Environ. Microbiol. 16: 2157-2167.

19. Lugtenberg B, Kamilova F. 2009. Plant-growth-promoting rhizobacteria. Annu. Rev. Microbiol. 63: 541-556.

20. Berendsen RL, Pieterse CMJ, Bakker PAHM. 2012. The rhizosphere microbiome and plant health. Trends Plant Sci. 17: 478-486

21. Berg G, Grube M, Schloter M, Smalla K. 2014. Unraveling the plant nnicrobiome: looking back and future perspectives. Front. Microbiol. 5: 148

22. Li YC, Yang TY, Zhang PP, Zou AL, Peng X, Wang LL, et al. 2012. Differential responses of the diazotrophic community to aluminum-tolerant and aluminum-sensitive soybean genotypes in acidic soil. Eur. J. Soil Biol. 53: 76-85.

23. Yang T, Ding Y, Zhu Y, Li Y, Wang X, Yang R, et al. 2012. Rhizosphere bacteria induced by aluminum-tolerant and aluminumsensitive soybeans in acid soil. Plant Soil Environ. 58: 262-267.

24. Li YL, Fan XR, Shen QR. 2008. The relationship between rhizosphere nitrification and nitrogen-use efficiency in rice plants. Plant Cell Environ. 31: 73-85.

25. Inceoglu O, Salles JF, van Overbeek L, van Elsas JD. 2010. Effects of plant genotype and growth stage on the betaproteobacterial communities associated with different potato cultivars in two fields. Appl. Environ. Microbiol. 76: 3675-3684.

26. Lu GH, Tang CY, Hua XM, Cheng J, Wang GH, Zhu YL, et al. 2018. Effects of an EPSPS-transgenic soybean line ZUTS31 on rootassociated bacterial communities during field growth. PLoS One 13: e0192008.

27. Wen ZL, Yang MK, Du MH, Zhong ZZ, Lu YT, Wang GH, et al. 2019. Enrichments/Derichments of root-associated bacteria related to plant growth and nutrition caused by the growth of an EPSPS-Transgenic maize line in the field. Front. Microbiol. $10: 1335$.

28. Lu GH, Zhu YL, Kong LR, Cheng J, Tang CY, Hua XM, et al. 2017. Impact of a glyphosate-tolerant soybean line on the rhizobacteria, revealed by Illumina MiSeq. J. Microbiol. Biotechnol. 27: 561-572.

29. Kennedy K, Hall MW, Lynch MDJ, Moreno-Hagelsieb G, Neufeld JD. 2014. Evaluating bias of illumina-based bacterial 16S rRNA gene profiles. Appl. Environ. Microbiol. 80: 5717-5722.

30. Kozich JJ, Westcott SL, Baxter NT, Highlander SK, Schloss PD. 2013. Development of a dual-index sequencing strategy and curation pipeline for analyzing amplicon sequence data on the MiSeq Illumina sequencing platform. Appl. Environ. Microbiol. 79: 5112-5120.

31. Caporaso JG, Lauber CL, Walters WA, Berg-Lyons D, Huntley J, Fierer N, et al. 2012. Ultra-high-throughput microbial community analysis on the Illumina HiSeq and MiSeq platforms. ISME J. 6: 1621-1624.

32. Edgar RC. 2013. UPARSE: highly accurate OTU sequences from microbial amplicon reads. Nat. Methods 10: 996-998.

33. Edgar RC, Haas BJ, Clemente JC, Quince C, Knight R. 2011. UCHIME improves sensitivity and speed of chimera detection. Bioinformatics 27: 2194-2200.

34. Caporaso JG, Kuczynski J, Stombaugh J, Bittinger K, Bushman FD, Costello EK, et al. 2010. QIIME allows analysis of highthroughput community sequencing data. Nat. Methods 7: 335-336

35. Schloss PD, Westcott SL, Ryabin T, Hall JR, Hartmann M, Hollister EB, et al. 2009. Introducing mothur: open-source, platformindependent, community-supported software for describing and comparing microbial communities. Appl. Environ. Microbiol. 75: 7537-7541.

36. Bulgarelli D, Garrido-Oter R, Munch PC, Weiman A, Droge J, Pan Y, et al. 2015. Structure and function of the bacterial root microbiota in wild and domesticated barley. Cell Host Microbe 17: 392-403.

37. Zhou JZ, Wu LY, Deng Y, Zhi XY, Jiang YH, Tu QC, et al. 2011. Reproducibility and quantitation of amplicon sequencing-based detection. ISME J. 5: 1303-1313.

38. Edwards J, Johnson C, Santos-Medellin C, Lurie E, Podishetty NK, Bhatnagar S, et al. 2015. Structure, variation, and assembly of the root-associated microbiomes of rice. Proc. Natl. Acad. Sci. USA 112: E911-920.

39. Minerdi D, Fani R, Gallo R, Boarino A, Bonfante P. 2001. Nitrogen fixation genes in an endosymbiotic Burkholderia strain. Appl. Environ. Microbiol. 67: 725-732.

40. Griffith JC, Lee WG, Orlovich DA, Summerfield TC. 2017. Contrasting bacterial communities in two indigenous Chionochloa (Poaceae) grassland soils in New Zealand. PLoS One 12: e0179652. 
41. Huang SC, Wang XD, Liu X, He GH, Wu JC. 2018. Isolation, identification, and characterization of an aluminum-tolerant bacterium Burkholderia sp. SB1 from an acidic red soil. Pedosphere 28: 905-912.

42. Ash C, Priest FG, Collins MD. 1993. Molecular-identification of ribosomal-Rna group 3 Bacilli (Ash, Farrow, Wallbanks And Collins) using a Pcr probe test - proposal for the creation of a new genus Paenibacillus. Anton. Van Leeuwenhoek 64: 253-260.

43. Gardener BBM. 2004. Ecology of Bacillus and Paenibacillus spp. in agricultural systems. Phytopathology 94: 1252-1258.

44. Montes MJ, Mercade E, Bozal N, Guinea J. 2004. Paenibacillus antarcticus sp nov., a novel psychrotolerant organism from the Antarctic environment. Int. J. Syst. Evol. Microbiol. 54: 1521-1526.

45. Jie OY, Pei ZH, Lutwick L, Dalal S, Yang LY, Cassai N, et al. 2008. Paenibacillus thiaminolyticus: A new cause of human infection, inducing bacteremia in a patient on hemodialysis. Ann. Clin. Lab. Sci. 38: 393-400.

46. Berge O, Guinebretiere MH, Achouak W, Normand P, Heulin T. 2002. Paenibacillus graminis sp nov and Paenibacillus odorifer sp. nov., isolated from plant roots, soil and food. Int. J. Syst. Evol. Microbiol. 52: 607-616.

47. Valverde A, Peix A, Rivas R, Velazquez E, Salazar S, Santa-Regina I, et al. 2008. Paenibacillus castaneae sp nov., isolated from the phyllosphere of Castanea sativa Miller. Int. J. Syst. Evol. Microbiol. 58: 2560-2564.

48. Achouak W, Sutra L, Heulin T, Meyer JM, Fromin N, Degraeve S, et al. 2000. Pseudomonas brassicacearum sp nov and Pseudomonas thivervalensis sp. nov., two root-associated bacteria isolated from Brassica napus and Arabidopsis thaliana. Int. J. Syst. Evol. Microbiol. 50: $9-18$.

49. Belimov AA, Dodd IC, Safronova VI, Hontzeas N, Davies WJ. 2007. Pseudomonas brassicacearum strain Am3 containing 1aminocyclopropane-1-carboxylate deaminase can show both pathogenic and growth-promoting properties in its interaction with tomato. J. Exp. Bot. 58: 1485-1495.

50. Busse HJ. 2016. Review of the taxonomy of the genus Arthrobacter, emendation of the genus Arthrobacter sensu lato, proposal to reclassify selected species of the genus Arthrobacter in the novel genera Glutamicibacter gen. nov., Paeniglutamicibacter gen. nov., Pseudoglutamicibacter gen. nov., Paenarthrobacter gen. nov and Pseudarthrobacter gen. nov., and emended description of Arthrobacter roseus. Int. J. Syst. Evol. Microbiol. 66: 9-37.

51. Yan Y, Kuramae EE, de Hollander M, Klinkhamer PGL, van Veen JA. 2017. Functional traits dominate the diversity-related selection of bacterial communities in the rhizosphere. ISME J. 11: 56-66.

52. Apprill A, McNally S, Parsons R, Weber L. 2015. Minor revision to V4 region SSU rRNA 806R gene primer greatly increases detection of SAR11 bacterioplankton. Aquat. Microb. Ecol. 75: 129-137.

53. Parada AE, Needham DM, Fuhrman JA. 2016. Every base matters: assessing small subunit rRNA primers for marine microbiomes with mock communities, time series and global field samples. Environ. Microbiol. 18: 1403-1414.

54. Walters W, Hyde ER, Berg-Lyons D, Ackermann G, Humphrey G, Parada A, et al.2016. Improved bacterial 16S rRNA gene (V4 and V4-5) and fungal internal transcribed spacer marker gene primers for microbial community surveys. mSystems 22: e00009-15.

55. Snijders AM, Langley SA, Kim YM, Brislawn CJ, Noecker C, Zink EM, et al. 2017. Influence of early life exposure, host genetics and diet on the mouse gut microbiome and metabolome. Nat. Microbiol. 2: 16221. 